\title{
Info-gap decision theory and its potential applications in the clinic
}

"Info-gap decision theory ... provides a tool for evaluating both the robustness against pernicious uncertainty as well as the opportuneness from propitious uncertainty."

Personalized medicine seeks to match the innate characteristics of patients with potential interventions in order to secure the most favorable outcome whilst mitigating risk. Personalized medicine does not supplant the decisions made by healthcare professionals in conjunction with patients. In fact, ideally, the application of personalized medicine greatly augments the quality of that interaction by adding an objective measure of disease and response to the physician's professional judgment. In that context, the practitioner faces disparity between what is known and what needs to be known in order to realize the desired goal of a favorable outcome of treatment or an effective avoidance of disease or complication. Info-gap theory can help manage this challenge.

Physicians and patients both wish to choose a course of action with confidence, knowing that the consequence will be satisfactory to the patient. However, these two attributes of a treatment - success and reliability - are different and in conflict with each other. Ambitious goals may be risky. The crux of the challenge is uncertainty - fragmentary data and imperfect understanding. Choices that exhaustively exploit uncertain knowledge may tend to fall short of the quality of outcome, which is predicted by that knowledge, precisely because some of that knowledge is imprecise. On the other hand, better-than-anticipated outcomes are also possible when our knowledge is imperfect. Treatments must be selected, not only according to their predicted outcomes, but also according to the immunity of those outcomes to errors in the knowledge underlying the predictions and according to the opportunities inherent in the uncertainty. Info-gap decision theory [1] provides a tool for evaluating both the robustness against pernicious uncertainty as well as the opportuneness from propitious uncertainty. Info-gap theory is a methodology for decision-making under severe uncertainty, which has been applied in many disciplines [101].
Robustness trades off against quality

If our knowledge were complete and accurate, it would be reliable to select the treatment that is predicted to have the best outcome. However, as we have already explained, lacunae in information (info-gaps) prevent us from reliably identifying optimal outcomes. But why is the best estimate of the optimal outcome not necessarily also the best bet for an adequate outcome? How do we know which estimated outcome is most resistant (robust) to error in the knowledge upon which the estimate is based? How do we know which treatment to choose with the highest confidence of achieving a satisfactory result?

The answers to these questions depend on a deeper understanding of the conflict between the success of an outcome - its quality - and the immunity of that quality to uncertainty in the knowledge on which the quality is predicated.

The basic robustness theorem of info-gap theory is that the immunity of an outcome to uncertainty increases as the estimated quality of that outcome decreases. Robustness trades off against quality. One can talk of a cost of robustness - the increment in quality that must be sacrificed in exchange for a positive increment of robustness.

For example, consider a specific pharmaceutical intervention for the reduction of lowdensity lipoprotein (LDL) cholesterol [2]. Given demographic data and clinical studies combined with patient preferences among possible adverse side effects, one can estimate the quality of outcome of the intervention for a specific patient. However, the patient may not match the populations from which the data were taken. Equivalently, the patient may not have met the enrollment criteria for the study on which the intervention was based [3]. Furthermore, the patient may have no personal experience with the possible side effects, so his or her preferences may be quite uncertain. Small errors in any of these elements can result in a diminished

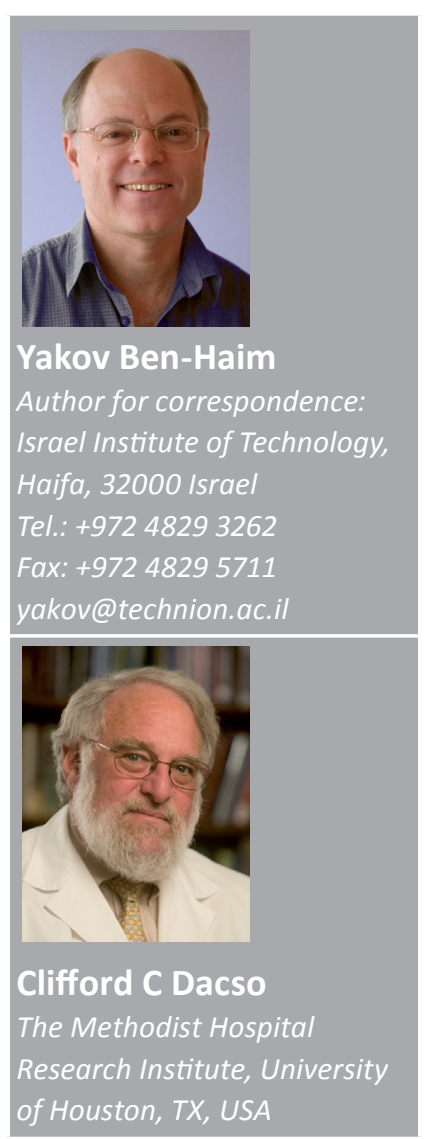

future medicine $^{\text {port }}$ fsg 
outcome satisfaction. Quality at a level that is somewhat less than predicted can be guaranteed, provided that the data and preferences do not err too much. The more the quality requirement is reduced, the greater the tolerance to error. The cost of robustness is the rate at which quality must be exchanged for immunity to error. Info-gap theory provides tools for quantitatively assessing this trade-off.

\section{"...lacunae in information (info-gaps) prevent us from reliably identifying optimal outcomes.'}

One possible outcome of considering the immunity to uncertainty is that we may actually reverse our preference among treatment options. For instance, consider two different treatments - two different therapies for pharmaceutical reduction of LDL cholesterol. Given the best estimates of clinical data and patient preference, one of these interventions will, in all likelihood, entail a better predicted outcome than the other. However, we already know that the preference between these treatments should not be based on their predicted outcomes, since these predictions have no robustness against info-gaps. How should we choose between these treatments? One way is for the patient to identify an acceptable or required 'critical' level of quality, and to then choose the treatment that is more robust to uncertainty at this critical quality. If the cost of robustness is the same for the two treatments, the more robust treatment will also be the preferred treatment based on the estimated outcome. But it can happen, nonetheless, that the treatment whose estimated outcome is better has a higher cost of robustness than the other treatment (recall that quality and robustness are distinct and conflicting attributes). Thus, the second treatment may be more robust than the first at the critical level of quality. In short, it can, and often does, happen that the highquality, low-robustness preference between the two treatments is different to the low-quality, high-robustness preference. Preferences can become reversed as the patient's needs and desires change.

\section{Opportuneness trades off against windfall}

Our knowledge is uncertain. The opportuneness of a treatment is a measure of how much the knowledge must deviate from reality in order for better-than-anticipated outcomes to be possible. Some treatments are very opportune: great windfalls are possible (although certainly not guaranteed) if the knowledge errs by only a small amount. Such treatments have a low cost of windfall. Other treatments have higher windfall cost, requiring a greater deviation of reality from prior knowledge in order for wonderful surprises to become possible.

Patients and physicians are usually, and rightfully, risk averse, and pay far more attention to robustness than to opportuneness. Nonetheless, opportuneness is sometimes useful, for instance, as the deciding vote between two treatments whose robustness is similar at the required outcome.

There are potential applications of info-gap theory in clinical practice. We have already mentioned the application of info-gap robustness and opportuneness decision strategies for choosing pharmaceutical intervention in the management of LDL cholesterol [2]. Similar analyses are possible for other clinical decisions that are accompanied by severe uncertainty in the clinical data, the state of the patient or the patient's preferences towards possible adverse outcomes. Examples include the choice of therapy for prostate cancer and the selection of an intervention for care of diabetes [4].

\section{"...immunity to uncertainty of an outcome increases as the estimated quality of that outcome decreases."}

There are other applications of info-gap theory, such as individualizing clinical guidelines based on uncertain asymmetric information about a patient. Consider a series of clinical tests characterizing a patient's condition. For example, the physician may assess cardiac risk with factors as diverse as allelic variants of USF1 [5] and waist:hip ratio. Diagnostic regressions based on demographic data specify the probability of a cardiac event with various interventions. Standard procedure would have the physician use these regressions and professional judgment to select the intervention whose predicted probability is minimal.

However, now suppose that the degree to which the patient actually fits the demographic profile upon which the guideline is based is uncertain. This means that selecting an intervention based on the predicted outcome is unreliable, since these predictions have no robustness to uncertainty.

In addition, suppose that the physician knows that the patient is a male long-distance runner whose high-density lipoprotein (HDL) 
cholesterol level should be quite high while, in fact, it is at the lower range of normal. That is, the physician has uncertain asymmetric information - this patient's HDL should be higher than demographically normal, although we do not know how much higher. The patient's HDL level is 'abnormally normal', but we cannot calibrate this abnormality owing to the lack of information about this patient or the subgroup to which he belongs. This asymmetric information is based on the contextual understanding of the physician - it is only very weakly quantitative and it is highly uncertain (we do not know how large a deviation is normal for this patient). Nonetheless, it is valuable in adapting standardized guidelines for this particular case.

\section{"...info-gap theory has many potential applications in personalizing medical art and technology."}

An info-gap robustness analysis of this asymmetric information can reveal the tradeoff between robustness to the uncertain idiosyncracy of this patient and the quality of outcome for different interventions. The costs of robustness for different interventions can be assessed and the possibility of reversal of preference between interventions can be explored with salutary results for the patient.

Info-gap theory can augment clinical safety. Clinical procedures often entail numerous serial and parallel steps with complex interdependencies. Consider blood transfusion. A partial list of the actions involved in transfusion includes patient preparation, blood sampling, typing the blood sample, recording the measured blood type attributes, transmitting these data to the blood bank, initiating the transfusion and supervising the patient's response. Errors at some stages can have severe adverse effects, which any hospital takes great efforts to avoid. How reliable (or risky) is a specific procedure with specified redundancies (e.g., of label verification) or personnel requirements (e.g., registered nurse or physician involvement) or mechanization (e.g., bar-coded labels)? Where in the procedure are resources most efficiently allocated to lower the risk? What uncertainties limit our ability to answer these questions?

Probably the most important uncertainties are the unknown and variable correlations among possible errors at different stages. All errors are rare, so the assumption of statistical independence results in a very low estimated risk because independent probabilities are multiplied together. However, correlated errors can arise by various mechanisms. First, interpersonal factors, such as mutual trust and professional admiration, can actually detract from the independence of human redundancy. Second, highly elaborate safety measures can induce local negligence at multiple points in the process. Third, the very success of the safety measures - low rates of failure - can induce a system-wide relaxation of vigilance with consequent correlated failures. The unknown correlations among purportedly independent rare errors are info-gaps that can vastly alter the actual risk. Since many errors fortunately do not result in pathological outcomes, measured over-all error rates are probably under-estimates of actual error rates. An info-gap analysis of robustness to uncertain correlations between errors can identify more vulnerable stages in the procedure. A robustness analysis can augment the confidence in estimates of risk, and can improve the allocation of resources for reducing risk.

In conclusion, info-gap theory has many potential applications in personalizing medical art and technology.

\section{Financial \& competing interests disclosure}

The authors have no relevant affliations or financial involvement with any organization or entity with a financial interest in or financial conflict with the subject matter or materials discussed in the manuscript. This includes employment, consultancies, honoraria, stock ownership or options, expert testimony, grants or patents received or pending, or royalties.

No writing assistance was utilized in the production of this manuscript.

\section{Bibliography}

1 Ben-Haim Y: Info-Gap Decision Theory: Decisions Under Severe Uncertainty (2nd Edition). Academic Press, London, UK (2006).

2 Ben-Haim Y, Dacso CC, Carrasco J, Rajan N: Heterogeneous uncertainties in cholesterol management. Int. J. Approx. Reason. 50, 1046-1065 (2009).
Greenfield S, Kravitz R, Duan N, Kaplan SH: Heterogeneity of treatment effects: implications for guidelines, payment, and quality assessment. Am. J. Med. 120 (4 Suppl. 1), S3-S9 (2007).

4 Ben-Haim Y, Zacksenhouse M, Keren C, Dacso CC: Do we know how to set decision thresholds for diabetes? Med. Hypotheses 73, 189-193 (2009).
5 Komulainen K, Alanne M, Auro K et al.: Risk alleles of USF1 gene predict cardiovascular disease of women in two prospective studies. PLoS Genet. 2(5), 672-681 (2006).

\section{Website}

101 Info-gap decision theory http://info-gap.com 